\title{
L'ENSILAGE ET LA PRODUCTION FROMAGÈRE ET BEURRIÈRE EN SUISSE* (suite et fin)
}

\author{
par le Dr W. DORNER \\ Ingénieur agronome
}

Attaché à l'établissement fédéral d'industrie laitière et de bactériologie à Liebefeld. Berne. Chef : Mr. le prof. Dr. R. Burri

\section{a). Provenance de l'agent du gonflement.}

Il s'agissait d'établir tout d'abord si l'affouragement au fourrage ensilé était vraiment la cause du gonflement du fromage. Les essais y relatifs ont été entrepris sous la direction de Kü RSTEINER (11) à Bürglen, Witzwil et Liebefeld. Les résultats obtenus à Bürglen étant particulièrement intéressants, nous allons leur consacrer quelques mots. Les essais de contrôle qui sont nécessaires pour avoir une base de comparaison n'ont pu dans la plupart des cas être faits simultanément avec l'essai prinsipal. La fabrication a été contrôlée pendant une certaine période, avant qu'ait commencé l'affouragement de l'herbe ensilée et après eəlui-ci. Le contrôle s'est étendu surtout à la qualité du lait, la fabrication (température et temps du chauffage, etc.) et la présure. Une fois l'affouragement du fourrage ensilé bien en train, il a été procédé à l'essai principal. La fabrication a été conduite suivant les mêmes règles que lors de l'essai de contrôle. La présure employée, grâce à une étuve pour présure et à la culture de fromagerie, était régulièrement de la même qualité que pendant l'affouragement à sec. Le contrôle du lait dans les deux essais n'a pas révélé de différences appréciables. Les conditions nécessaires pour donner du poids et rendre les résultats obtenus indiscutables, étaient donc remplies.

Il faut encore ajouter que dans l'essai principal les fromages ont été fabriqués avec 130-180 litres de lait provenant de l'étable dans laquelle les bêtes reçoivent de l'herbe ensilée, mêlés à environ 900 litres de lait ordinaire provenant des autres fournisseurs.

Voyons les résultats maintenant. Tout d'abord les fromages de l'essai principal semblaient vouloir ressembler à ceux de l'essai de contrôle, mais bientôt, au bout d'une dizaine de jours, ils se mirent à gonfler. Les résultats seront mieux illustrés, si nous indiquons les notes obtenues à la taxation par les experts. Les fromages fabriqués du 20 décembre 1917 au 30 janvier 1918 (période de contrôle) ont été tous taxés comme qualité $1 a$ ou $1 b$. Les fromages du 4 janvier au 28 février (période de l'affouragement à l'herbe ensilée) ont donné les résultats suivants :

2 pièces de qualité $\mathrm{I} b, 21$ pièces de qualité II.

\footnotetext{
* Les clichés des figures 1 et 3-8 ont été mis aimablement à notre dispcsition par l'Administraticn de la revue "Schweiz. landw. Monatshefte".
} 
1 pièce de qualité II-III, 22 pièces de qualité III.

2 pièces de qualité III-IV, 10 pièces de qualité IV.

Les fromages du 1 er au 12 mars (période de contrôle final) ont tous été taxés comme qualité I $a$ ou $\mathrm{I} b$, à l'exception de celui du 3 mars, qui a reçu la note II. La taxation a été faite d'après l'échelle du $\mathrm{D}^{\mathrm{r}}$ Wyssmann, que nous caractérisons ci-après.

Qualité $I V$. - Le fromage est défectueux à tous les points de vue;

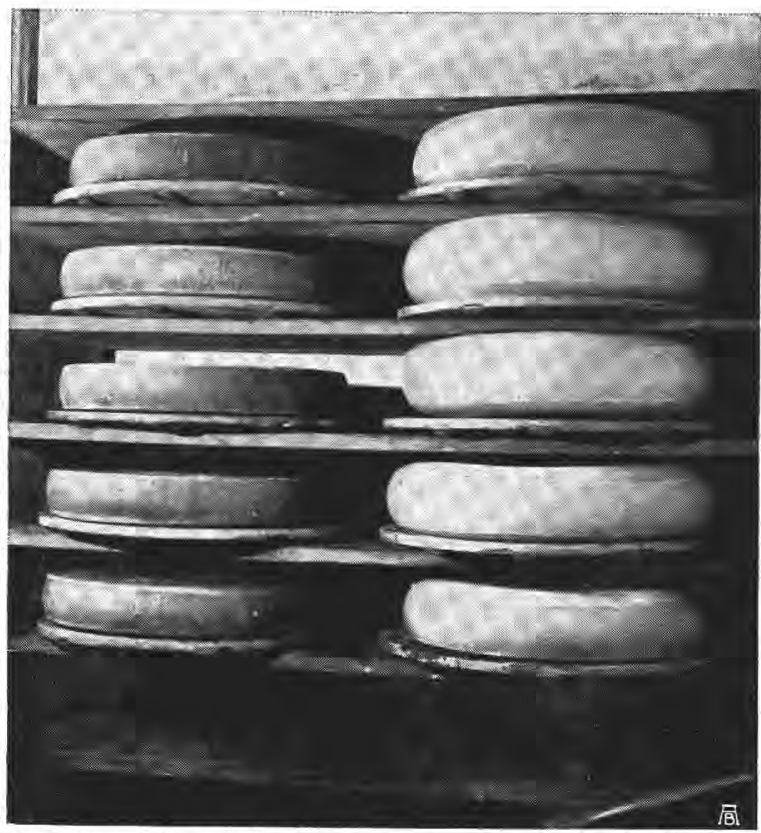

Fig. 3. - A gauche fromages normaux, à droite fromages gonflés comme suite à l'alimentation des vaches au fourrage ensilé. - Phot. Dr. W. Staub.

il a mauvais goût, mauvaise odeur, vilaine ouverture, une pâte défectueuse et finalement, il estfendu. En sondant le fromage, il s'échappe un gaz combustible del'ouverture faite par la sonde. Le fromage estfortement gonflé; sa pâte esit spongieuse ; l'ouverture rappelle les pores d'une éponge, ou si le fromage s'est affaissé, les ouvertures que l'on aperçoit dans une tête de choux coupée en deux.

Qualité $I I I$. - Le fromage n'est pas encore fendu. Il a les autres défauts de la IVe qualité, mais ceux-ci sont moins prononeés.

Qualité II. - Le fromage est gonflé, pas encore fendu, mais le goût et l'odeur sont meilleurs.

Qualité $I$ b. - Odeur et goût bon, pâte un peu ferme; ouverture en général bonne, de temps en temps un peu de lainure.

Qualité Ia. - Odeur, goût, ouverture et pâte, tout est normal.

Cet essai prouve abondamment que le fourrage ensilé donné aux vaches occasionne le gonflement du fromage d'Emmental. Il s'agissait ensuite de voir si, comme il semblait probable, l'agent du gonflement du fromage était contenu dans le fourrage ensilé. De nombreuses analyses bactériologiques ont établi qu'il en était ainsi. Cependant on rencontre 
de très grandes différences entre les diverses parties d'un échantillon. Tandis que certaines parties ne contiennent pas de bacilles butyriques (bac. amylobacter), d'autres en contiennent bien des millions par gramme de fourrage. Cette répartition inégale des bacilles dangereux dans le fourrage rend les résultats de l'analyse bactériologique de celui-ci incertains.

\section{b). La voie que suivent les bacilles pour parvenir dans le lait.}

Le fourrage ensilé contenant des bacilles butyriques, la première chose à faire était de voir si ces microbes se retrouvent dans les excréments. En effet, si les spores des bacilles butyriques ne sont pas détruites pendant la dịgestion elles doivent se retrouverdans les fèces. Par la digestion dufourrage ensilé il se réalise un mélange intime de la portion affouragée, mélange

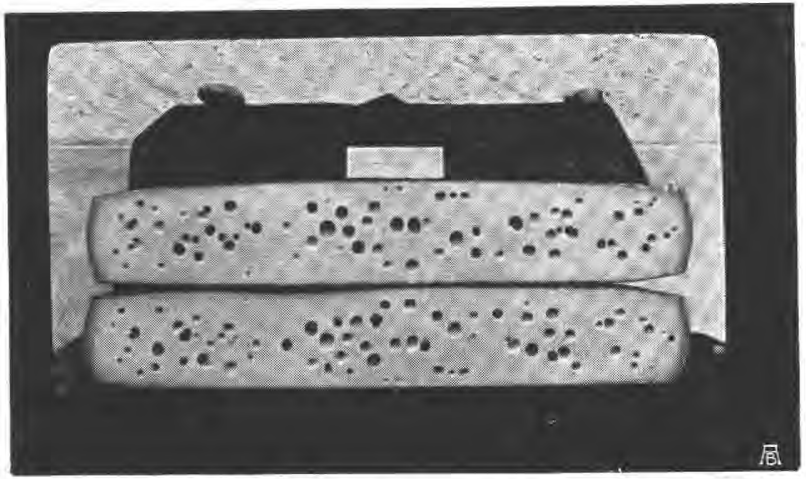

Fig. 4. - Section d'un Emmental de première qualité. Phot. Dr. W. Staub. que l'on ne pourrait pratiquement pas effectuer d'une manière aussi parfaite. Comme il ressort d'un travail de BuRRI, STAub et HoHL (12), l'essai a prouvé le bien fondé de cette idée. Les fèces des animaux alimentés au fourrage ensilé contiennent de grandés quantités de spores dangereuses. Pour illustrer nos dires, il suffit de citer une seule d'entre les séries d'essais effectués par notre établissement. La série a été conduite parallèlement à un essai d'affouragement effectué au Liebefeld. Dans la période du 21 février au 18 avril 1919, l'analyse bactériologique des fèces des animaux a été faite presque journellement. Nous ne voulons pas fatiguer nos lecteurs en leur donnant les résultats détaillés, les moyennes des différentes périodes suffiront.

Dans la période de contrôle préalable, du 21 au 28 février, où les animaux recevaient la nourriture habituelle, les fèces contenaient en moyenne 460 spores de bac. butyriques par gramme. Dans la période transitoire du 28 février au 7 mars, où les animaux recevaient des doses croissantes de fourrage ensilé, le nombre de spores de bac. butyriques par gramme de fèces s'élevait en moyenne à 56,200. Dans la période d'essai principal du 7 mars au 4 avril, où les animaux recevaient les rations maximales de fourrage ensilé, la teneur des fèces s'élevaient en 
moyenne à 470,000 spores par gramme. Le nombre maximum de spores constaté fut $2,750,000$ le 31 mars. Dans la période des rations décroissantes, du 4 au 11 avril, la moyenne était de 17,300 spores par gramme, tandis que dans la période de contrôle finale, où le bétail ne recevait plus de fourrage ensilé, période qui a duré du 11 au 18 avril, les fèces ne contenaient plus que 150 spores de bac. butyriques par gramme.

Ces chiffres parlent d'eux-mêmes. Tandis qu'il n'a pas été toujours possible de dézeler les bac. butyriques dans le fourrage ensilé, les fèces

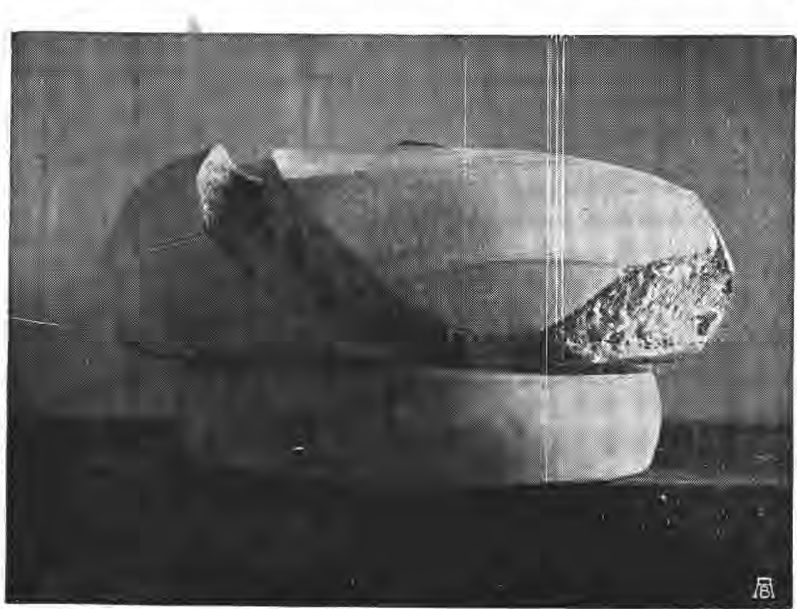

Fig. 5. - En bas fromage témoin fait de lait normal, en haut fromage éclaté par suite du gonflement provoqué par le bac. amylobacter du fourrage ensilé. - Phot. Dr. W. Staub.

des animaux quien reçoivent des portions relativement modestes ont toujours une teneur en bacilles butyriques distinctement plus élevée que celle des excréments d'animaux nourris de la façon ordinaire.

Une constatation accessoire intéressante qui découle de cetessai est que l'analyse bactériologique des fèces des animaux qui reçoivent du fourrage ensilé est le moyen le plus sûr et le plus simple pour déceler la présence du bacille amylobacter dans le fourrage ensilé. Le bacille amylobacter se trouvant en grand nombre dans les excréments, il n'est pas étonnant qu'il se trouve aussi dans le lait. En effet, on sait que les vaches se salissent presque toujours avec leurs excréments. Ces derniers sèchent sur le pelage de l'animal et tombent dans le lait sous forme de poussières. Le bacille butyrique peut également passer directement du fourrage dans le lait. L'observation suivante en est la preuve : Kürsteiner (13), appelé dans une fromagerie où ]'on fabrique du fromage de Spalen, découvre que le gonflement constaté sur les fromages ne provient pas de l'affouragement d'herbe ensilée. Un agriculteur ayant essayé d'ensiler de l'herbe et l'ensilage ayant mal réussi, il a utilisé ce fourrage comme litière. Les bacilles butyriques contenus dans eette litière s'attachent aux flancs des vaches et passent dans l'air de l'étable, De là ils tombent dans le lait. 


\section{c). Possibilité d'empêcher l'infection du lait par le bacille amylo- bacter du fourrage.}

On avait deux moyens pour éviter l'infection du lait par le bac. amylobacter. D'une part on pouvait essayer d'entraver son développement dans le silo ou l'empêcher d'y pénétrer et d'autre part on pouvait prendre des mesures pour protéger le lait de l'infection provenant du fourrage. Les deux voies ont été expérimentées. KüRSTEINER (14) a d'abord essayé d'empêsher le bacille amylobacter de se développer dans le silo. L'addition de 1,25 pour mille de sel de cuisine à l'herbe au moment de l'ensilage n'a pas donné de résultat satisfaisant. Dans un essai fait en petit, l'herbe avait été additionnée de $5 \%$ de sel sans que le fourrage ensilé soit indemne de bacilles butyriques. L'addition de 1 litre de culture de fromagerie par 100 kilos d'herbe à l'ensilage n'a pas donné de résultat satisfaisant non plus.

Dans le même

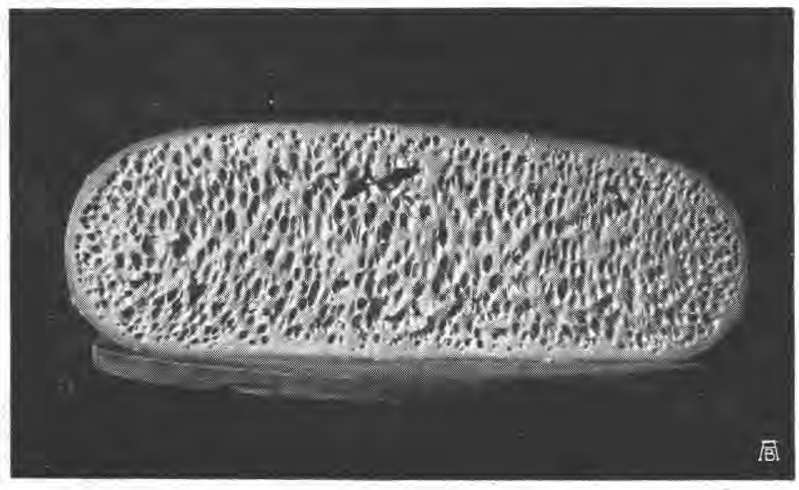

Fig. 6. - Section d'un fromáge gonflé par le bac. amylobacter. Phot. Dr. W. Staub.

travail K $\ddot{\mathrm{U}} \mathrm{RS}-$

TEINER (14) étudie la question, pourquoi les bacilles butyriques sont inégalement répartis dans le fourrage ensilé.

Kürsteiner et Staub ont émis l'hypothèse que les particules de terre introduites dans le silo avec l'herbe pourraient bien être la cause principale de l'infection du silo avec le bacille amylobacter. Les particules de terre seraient alors les centres de développement du bacille butyrique. Il suffit alors d'admettre que nos microbes se développent surtout sur place pour expliquer leur répartition inégale dans le fourrage ensilé. Cette hypothèse est rendue probable par les essais de Hohl, qui a prouvé que plus le fourrage ensilé était contaminé de terre, plus il était riche en bacilles butyriques. La question : "Est-il possible de réduire par l'exclusion de la source d'infection (la terre) le nombre des basilles butyriques du fourrage assez fortement pour permettre une fabrication normale du lait produit ? " fut alors mise à l'étude. Kürsteiner - et Staub y répondent négativement. Sous la direction du Dr Schmid, administrateur des stations fédérales d'essais agricoles, un des silos d'essais de Liebefeld a été rempli d'herbe, en prenant toutes les précau- 
tions qu'il serait possible de prendre dans la pratique pour réduire la quantité de terre adhérant à l'herbe à un minimum. Par ces mesures il a été possible de réduire le nombre de bacilles butyriques du fourrage de telle sorte, que les excréments du bétail alimenté avec celui-ci ne contenaient en moyenne que 900,000 spores du bacille butyrique par gramme, tandis que les excréments contenaient lors de l'affouragement de l'herbe ensilée, préparée sans précautions spéciales, 2,910,000 spores par gramme. Cependant, les fromages fabriqués alors que les fèces contenaient 900,000 spores de bacilles butyriques par gramme ont tout aussi bien gonflé que les fromages de contrôle lors de l'affouragement à l'herbe ensilée ordinaire.

Avec Kürsteiner et Staub (15) nous avons examiné s'il était possible d'éliminer les sources d'infection lors de la traite et d'obtenir un lait propre à la fabrication du fromage d'Emmental, malgré l'affouragement d'herbe ensilée. D'entente aveo M. le Dr Schmid, nous avons surveillé personnellement la traite de dix vaches recevant du fourrage ensilé préparé très soigneusement, en évitant autant que possible l'infection par la terre. La traite avait lieu comme suit : Celui des deux vachers chargé de la traite met, matin et soir, un pantalon et une blouse propres. Avant la traite de chaque vache il se lave les mains soigneusement. Son camarade l'aide, en nettoyant avec soin chaque mamelle au moyen de deux linges stérilisés et ce dernier aussi se lave les mains à chaque nouvelle vache. L'étable est nettoyée avec le plus grand soin et la queue des vaches est attachée pendant la traite. Le lait est recueilli dans un

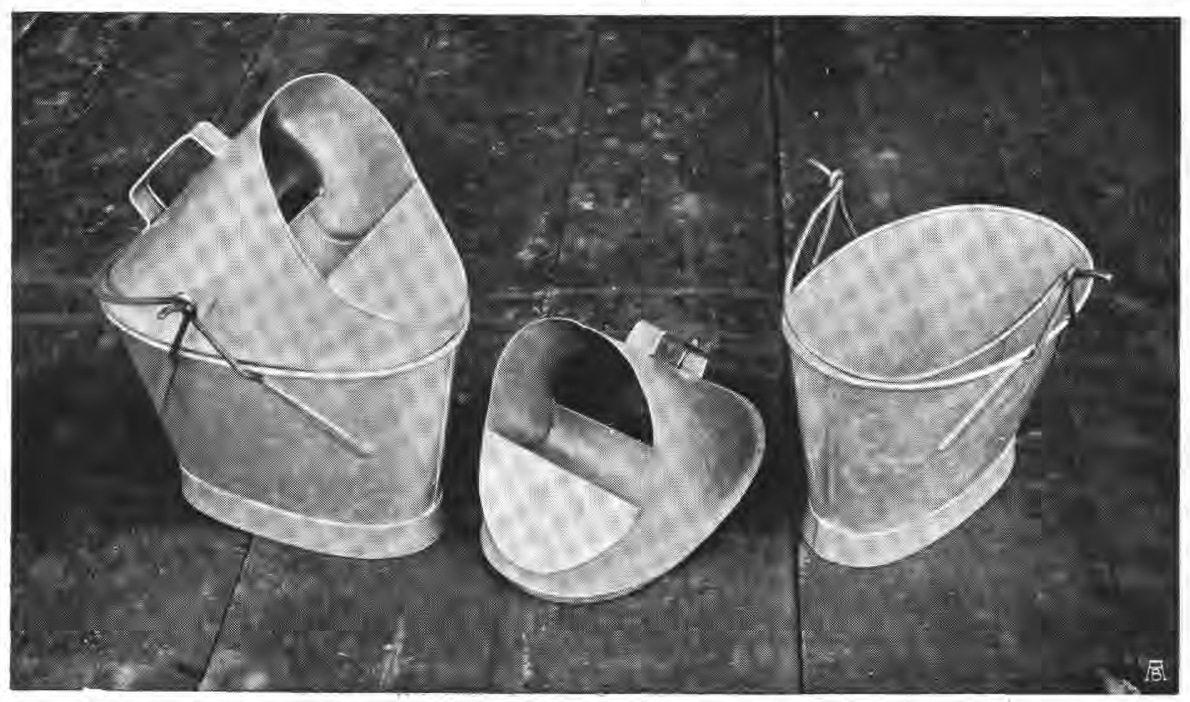

Fig. \%. - Seau à traire spécial, modèle Dr. Sprecher, utilisé pour la production de laít très propre. Phot. Dr. W. Staub. 
seau à traire muni d'un dispositif spécial qui empêche les poils et la poussière de tomber dans le lait. De cette façon nous sommes arrivés à avoir trois à six fois moins de microbes dans notre lait que dans celui obtenu des mêmes vaches sans prendre de précautions spéciales. La teneur moyenne en bactéries du lait trait de cette manière était de 850 par $\mathrm{cm}^{3}$, la numération étant faite sur plaques de gélatine au petit lait peptoné. Il est clair que dans la pratique des soins de propreté aussi minutieux ne sont pas réalisables. Les fromages fabriqués avec ce lait portaient tous les marques caractéristiques de l'affouragement avec l'herbe ensilée.

Conclusion : Il est impossible de fabriquer du fromage d'Emmental avec le lait produit lors de l'affouragement d'herbe ensilée, même lorsque l'ensilage a lieu avec un soin tout spécial et que l'on prend toutes les mesures de propreté pour empêcher une infection lors de la traite.

\section{d) Nombre de bacilles butyriques nécessaires pour faire gonfler le fromage.}

Le résultat de l'ex périence que nous venons de relater faisait présumer qu'un très petit nombre de bacilles butyriques devait suffire pour faire gonfler le fromage. Il était cependant d'un certain intérêt d'avoir des données précises sur le nombre de bacilles nécessaires pour provoquer le gonflement du fromage. En même temps on pouvait fournir la preuve absolue que le bacille amylobacter est vraiment l'agent du gonflement des fromages. Cette preuve, pour ainsi dire la clé de voûte de toute la série d'essais, a été donnée par KürsteIner (16). A cet effet il a ensemencé un lait normal de différentes quantités de bacilles butyriques, pour dézouvrir combien de ces bacilles le lait doit contenir pour que le fromage présente le gonflement caractéristique qui se produit lorsque les vaches reçoivent du fourrage ensilé. Connaissant le nombre des bacilles additionnés au lait, il a calculé le nombre de bacilles se trouvant dans un kilo de fromage frais, en admettant que tous les bacilles ajouté passent dans le fromage et que 10 litres de lait donnent 1 kilo de fromage frais. Voici les résultats obtenus :

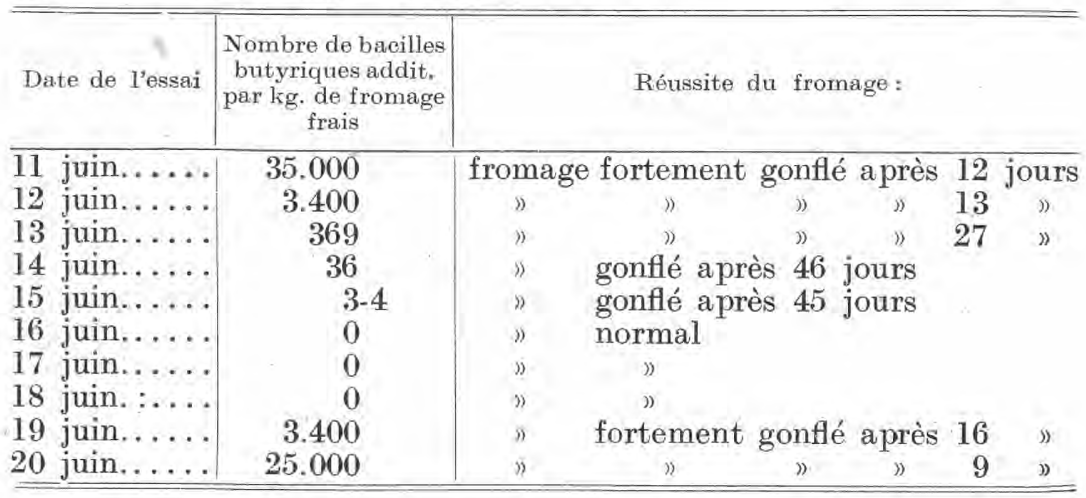


Il est peu d'essais aussi probants que celui-ci. Les chiffres parlent d'eux-mêmes. Trois bacilles butyriques pour 10 litres de lait suffisent pour faire gonfler le fromage !

Il y a lieu de rappeler ici ce que nous avons dit au sujet du développement du bac. amylobacter dans les milieux de culture du laboratoire. Seuls quelques pour mille se développent. Quoi qu'il en soit, l'expérience de Kürsteiner démontre formellement la grande importance de la quantité de bacilles qui s'infiltrent dans le lait. Plus il y en a, plus le gonflement est violent et rapide.

\section{e) Manière de déceler le lait produit avec du fourrage ensilé.}

Dès le moment où notre établissement s'est occupé de la question de l'ensilage, on s'est efforcé de trouver un moyen pour déceler le lait produit avec du fourrage ensilé. Toutes les méthodes d'analyse usuelles, lactofermentateur, réductase, etc.s e sont révélées insuffisantes L'essai du lactofermentateur à $30^{\circ}$ précédé de la pasteurisation du lait à éprouver pendant 10 minutes à $80^{\circ}$ n'a pas donné de bons résultats non plus, parce qu'un bacille anaérobie très fréquent, mais non dangereux, le bac. saccharobutyricus immobilis, Grassberger et Schattenfroh, se développe très souvent dans le lait pasteurisé et que d'autre part toutes les souches du bac. amylobacter ne peuvent pas y prospérer. Il n'a pas été possible de trouver une méthode satisfaisante pour reconnaître le lait produit avee le fourrage ensilé. On peut, il est vrai, ensemencer le lait suspect dans de la gélose glucosée. Si la pollution du lait est grave, on trouvera jusqu'à 100 bacilles butyriques par $\mathrm{cm}^{3}$. Si le lait est produit avec certains soins, on n'y trouvera pas l'agent de gonflement, malgré que le lait soit absolument impropre à la fabrication du fromage. Un moyen efficace de contrôle du lait manque complètement. Par contre le contrôle bactériologique des excréments des vaches donne d'excellentes indications sur la qualité du lait. On risque le gonflement du fromage en cave sitôt que la teneur des fèces atteint 1.000 bacilles butyriques par gramme.

\section{f) Peut-on entraver le gonflement du fromage en cave ?}

Il ressort d'une publication de KürSTEINER (14) qu'il est possible de fabriquer des fromages maigres avec le lait de vaches alimentées au fourrage ensilé. Des essais faits en séries à. Bürglen et à Uttewil ont prouvé qu'on obtenait d'excellents fromages maigres avec ce lait. On a présumé tout d'abord que l'agent du gonflement passait dans la crème et que c'est de cette manière que s'explique cette réussite. L'expérience a prouvé qu'il en était autrement.

Une série de fromages mi-gras fa briqués à Bürglen avec du lait normal entier, additionné de lait centrifugé provenant de l'exploitation qui 
affourageait de l'herbe ensilée, ont gonflé de façon typique. Le nonfondé de cette hypothèse étant établi, les résultats différents obtenus avec la fabrication de fromage maigre et de fromage gras ne pouvaient provenir que de la fabrication différente.

Une autre hypothèse, qui explique la réussite différente des fromages gras et maigres préparés avec le lait de vaches nourries à l'ensilage, par la technique différente que l'on emploie dans la fabrication de ees deux sortes, s'est trouvée juste.

KÜRsteINER a fait l'essai de fabriquer des fromages d'Emmental, donc des fromages gras, à la façon des maigres, avec du lait produit avec du fourrage ensilé. Les fromages n'ont été chauffés qu'è, $37^{\circ} \mathrm{C}$. Aucun n'a gonflé.

$\mathrm{Vu}$ qu'il est possible d'empêcher le boursouflement des fromages gras en les fabriquant comme les maigres, il devait être possible de faire gonfler des fromages maigres en les chauffant comme les gras. L'essai a confirmé cette idée. Des fromages maigres, chauffés à 52-54ㅇ C., ont gonflé.

On ne sait pas encore si c'est la haute température à laquelle on chauffe les fromages gras en soi, si c'est la consistance plus ferme de la pâte qui en résulte ou encore si c'est pour une autre raison que les fromages d'Emmental se boursouflent.

Bien que les fromages d'Emmental, fabriqués à la mode des maigres, n'aient pas gonflé et fussent parfaitement mangeables, ils ne répondaient cependant pas aux qualités que l'on exige d'un Emmental. Vu que c'est sous l'influence de la haute température à laquelle les Emmental sont fabriqués que ceux-ci se boursouflent, il est clair que tout autre fromage, fabriqué de façon analogue, subira le même sort. Les Gruyères gras et mi-gras, les Spalen, enfin tous les fromages gras à pâte cuite, ne peuvent pas être fabriqués avec du lait provenant d'étables dans lesquelles on emploie du fourrage ensilé.

Une modification de la fabrication dans le sens indiqué ne pouvait done pas entrer en ligne de compte pour la pratique. Il a fallu chercher ailleurs.

Le bacille amylobacter se trouvant dans le lait surtout sous la forme de spores (les spores résistent à une température de $80^{\circ}$ pendant au moins un quart d'heure), une pasteurisation du lait, sans porter atteinte à sa coagulabilité par la présure, devait fatalement rester sans résultat. Kürsteiner (17) en a cependant fait l'essai avec le résultat négatif auquel il fallait s'attendre. Dans le même travail Kürsteiner rapporte sur une expérience qui consistait à additionner le lait d'eau oxygénée, pour essayer de détruire les bacilles butyriques qu'il contenait. Le résultat a été mauvais. Le fromage avait un goût très désagréable.

L'addition de nitrate de soude au lait de fabrication étant susceptible, selon des recherches de Bokzhout et de VRiks, d'empêcher le bac. 
amylobacter de se développer dans le fromage d'Edam, nous expérimentons actuellement ce procédé pour l'Emmental. Il n'est pas possible de dire si l'addition d'une certaine quantité de salpêtre remplira son but ou non.

Voyons maintenant ce qu'il en est des deux autres sortes de fourrage ensilé qui ont été expérimentées en Suisse. Quelques mots suffiront, étant donné que tout ce qui a trait aux méthodes pour l'ensilage doux s'applique également aux autres sortes de fourrage ensilé. Le fourrage ensilé à l'électricité n'est pas moins dangereux que le fourrage ensilé doux. Kürsteiner (18) donne des analyses bactériologiques des excréments de vaches alimentées à l'herbe ensilée électriquement; à Wangen les excréments contenaient de 100.000 à 1.000 .000 de spores de bacilles butyriques.

Les résultats obtenus à Liebefeld ressortent du tableau ci-dessous :

\section{Alimentation}

Ration sans fourrage ensilé. . . . . . . . . . Rations croissantes d'herbe ensilée électriquement. Rations maximales d'herbe ensilée électriquement. Rations décroissantes d'herbe ensilée électriquement . Ration sans fourrage ensilé. . . . . . . . . . .
Nombre moyen de spores de bac. amylobacter par gramme d'excréments

300

92.500

190,785

20.350

67

Ces quelques chiffres démontrent à l'évidence que pas plus que l'ensilage doux, l'ensilage électrique ne saurait être utilisé pour produire un bon lait de fromagerie.

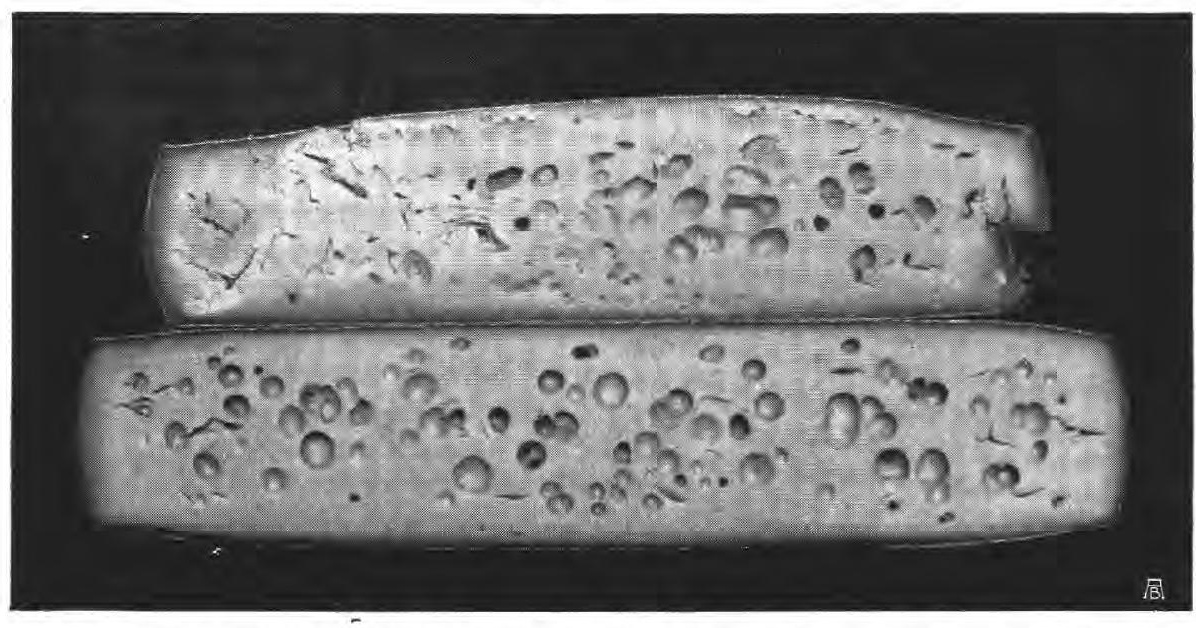

Fig. 8. - Le meilleur (en bas) et le plus mauvais (en haut) fromages fabriqués en 1921 à Witzavil lorsque les vaches recevaient du maïs ensilé. - Phot. Dr. W. Staub. 
Pour le maïs ensilé la question se complique. Si le maïs est ensilé correctement, on obtient des résultats favorables. Si par contre on ensile du maïs trop peu mûr ou que l'on commet d'autres fautes à l'ensilage, on obtient un fourrage des plus dangereux. Etant donné cela, l'ensilage du maïs doit être considéré comme dangereux pour la fabrication du fromage d'Emmental.

L'ensilage de tournesol et de topinambour a donné de très mauvais résultats. Le tableau suivant, tiré d'un travail de Kürsteiner (2), donne les résultats de l'analyse des excréments.

Date

Alimentation

7-21 nov.

21-24 "

21-24 ")

24-30

jusq. 3 déc.

3- 9 déc.

9-12 》)

$12-18$ "

18-21

$21-27$

Pas de fourrage ensilé ensilé ensilé bour ensilé
Rations croissantes de tournesol

Rations croissantes de tournesol

Jusqu'à $20 \mathrm{~kg}$. de tournesol ensilé par tête et par jour

Nombre moyen de spores de bac. amylohacter par gramme d'excréments $0-100$

$100.000-1.000 .000$

Rations décroissantes de tournesol

$10.000-1.000 .000$

$1.000-10.000$

1.000 .000

1.000 .000 ensilé par tête et par jour

Rations décroissantes de topinam-

Pas de fourrage ensilé
$10-10.000$

Il est évident qu'avec des fèces aussi riches en bacilles butyriques on ne saurait produire un lait susceptible d'être transformé en fromage d'Emmental.

Essayons maintenant de résumer et de tirer les conclusions de ce qui précède :

Le fourrage ensilé doux contient en grand nombre des bacilles butyriques (bac. amylobacter $A$. M. et Bredemann), qui occasionnent le gonflement du fromage d'Emmental et sortes similaires, qui sont préparés avec le lait des vaches qui reçoivent ce fourrage. On ne connaît pour le moment aucun moyen pour prévenir ce gonflement. En conséquence, l'ensilage doux de l'herbe est impossible dans les régions où le lait sert à la préparation du fromage d'Emmental et de Gruyère, de Spalen et de sortes similaires chauffés à haute température pendant la fabrication. Il en est de même pour l'ensilage électrique, l'ensilage du tournesol et du topinambour. L'ensilage du maïs, bien qu'il donne de bons résultats dans certains 
cas, ne peut être recommandé non plus pour la production de lait de fromagerie.

Ce qui précède s'applique exclusivement aux fromages du genre Emmental ou Gruyère chauffés à plus de $44^{\circ} \mathrm{C}$. pendant la fabrication. Pour les autres sortes de fromage le risque de gonflement ensuite de l'action du bac. amylobacter ne paraît pas exister. C'est tout particulièrement le cas pour les pâtes molles si importantes en France. Cependant nous avons vu dans certains cas que des fromages de Tilsit gras préparés à trop haute température ont présenté un gonflement dû au bacille amylobacter du fourrage ensilé.

Pour la beurrerie, l'ensilage ne paraît pas jouer le rôle néfaste qu'il a pour la préparation des fromages à pâte dure. On prétend même, à tort ou à raison, que l'alimentation des vaches avec de l'herbe ensilée fait augmenter la teneur en graisse du lait. L'ensilage n'est cependant pas sans danger aucun pour la beurrerie. Chacun sait que le fourrage ensilé répand dans la règle une odeur forte spéciale. Or il est également bien connu que le lait, et plus spécialement sa matière grasse, prennent très facilement l'odeur des locaux dans lesquels ils séjournent. On risque done, lorsqu'on donne du fourrage ensilé aux vaches, que le beurre ait un goût d'ensilage désagréable. Ce défaut est cependant facile à éviter. Il suffit de prendre certaines précautions pour empêcher l'air de l'étable de se charger de l'odeur d'ensilage au moment de la traite. A cet effet on évitera d'affourager l'ensilage pendant la traite ou de laisser le fourrage dans un local qui ne soit pas séparé de l'étable par des portes closes hermétiquement.

Les mêmes précautions sont à recommander lors de la production de lait pour la consommation en nature. Pour cette production l'ensilage est franchement avantageux. Le lait produit avec du fourrage ensilé est supérieur au lait produit ordinairement en hiver ensuite de sa teneur en vitamines. Cela est tellement vrai que certains médecins allemands exigent que le lait destiné aux bébés soit produit en hiver en alimentant les vaches au fourrage ensilé. En tous cas, des essais effectués dans un hôpital de Zurich ont démontré que le lait des vaches qui recevaient du fourrage ensilé était supporté sans inconvénients par les bébés.

\section{BIBLIOGRAPHIE.}

1. Dorner W. - L'ensilage du fourrage vert et la teneur des sols en bacilles butyriques. La Terre Vaudoise, 1924.

2. Kürtseiner J. - Die Emmentalerkäse-Qualitätsproduktion und das konservierte Grünfutter (Silofutter). Schweizerische Landwirtschaftliche Monatshefte, 1926, Nrn. 1-4.

3. Schatzmann R. - Alp-und Milchwirtschaftliche Monatsblätter 1833-1886.

4. GRAF M. - Das süsse Grünfutter, dessen Bereitung und Resultate.

5. WrRz J. - Die Süssgrünfutterbereitung, ein hervorragendes Mittel zur Steigerung der landwirtschaftlichen Produktion. Zürich 1918, Orell-Füssli. 
6. Voir à ce sujet : DoRner W. - L'ensilage du fourrage vert. Annuaire agricole de la Suisse, 1927, p. 295.

7. RUTGERS F. - Bericht über Versuche mit elektrischer Grünfutterkonservierung auf dem Gute Bocken bei Horgen. 1925, Maschinenfabrik Oerlikon.

8. Kuchier L. F. - Die ze:tgemässe Grünfutterkonservierung 1926, Datterer, Freising-München.

9. Burri R. - Zum Wiederaufleben der Pressfutterfrage. Schweiz Milchzeitung, 1918, Nr. 18.

10. DorNer W. - Beobachtungen über das Verhalten der Sporen und vegetativen Formen von Bac. amylobacter A.M. et Bredemann bei Nachweis- und Reinzuchtversuchen. Landw. Jahrbuch der Schweiz, 1924.

11. Kürsteiner J. - Ist Milch aus Ställen, in welchen den Kühen konserviertes Gras, sog. Süssgrünfutter, verabreicht wird, für die Emmentalerkäserei tauglich? Schweiz. Zentralblatt für Milchwirtschaft, 1918, Nos. 29-30.

12. Burri R., Staub W. und Hoнl J. - Süssgrünfutter und Buttersäurebazillen. Schweiz. Milehzeitung 1919, Nos. 78-83 und Schweiz. Zentralblatt für Milchwirtschaft, 1919, Nos. 40-43.

13. KÜRSTEINER J. - Ueber eine durch nachträgliche Blähung verursachte schwere Käserei-Betriebsstörung. Schweiz. Milchzeitung 1919, Nr. 3.

14. KürRsteiner J. - Zur Frage der Käsereitauglichkeit der Süssgrünfuttermilch. Schweiz. Milchzeitung 1919, Nos. 72-75 und Schweiz. Zentralblatt für Milchwirtschaft, 1919, Nos: 42-43.

15. Kürsteiner J., Staub W. und Dorner W. - Ist besonders reinlich gemolkene, aus konserviertem Gras erzeugte Milch für die Emmentalerkäserei tauglich ? Schweiz. Milchzeitung, 1922, Nr. 72.

16. Kürsteiner J. und StaUB W. - Ein weiterer Beitrag zur Abklärung der Süssgrünfutterfrage. Schweiz. Zentralblatt für Milchwirtschaft, 1. Dec. 1921.

17. Kǘrsteiner J. - Neuerdings aufgetretene, durch den Blähungserreger des Süssgrünfutters verursachte Käsereibetriebsstörungen, Schweiz. Zentral blatt für Milchwirtschaft, 1920.

18. Kürsteiner J. - Die heutige Graskonservierung, eine grosse Gefahr für die Emmentalerkäserei. Schweiz. Milchzeitung, 1922, Nos. 29-33.

\title{
L'ACTION DES HALOGËNES SUR LE LAIT ET SUR SES CONSTITUANTS
}

\author{
Par le Dr ALb-.J.-J. VANDEVELDE,
}

Professeur à l'Institut agronomique et à l'Université de l'Etat à Gand.

Les halogènes sont susceptibles d'agir sur le lait et sur ses constituants, comme antiseptiques, mais ils peuvent aussi agir chimiquement. A la suite de mes recherches sur les combinaisons halogénées des protides (1), j'ai été amené à étudier l'action des halogènes sur le lait et sur

(1) Protéines halogénées : I. Bromovalbumine, Rec. trav. chim. Pays-Bas, 1924, 43, 158. II. Bromof эptone, ibid., 1924, 43, 326. III. Bromogluten, ibid., 1924, 43, 706. IV. Bromofibrine, ibid., 1925, 44, 224. V. Bromocaséine, ibid., 1925, 44, 900. Halogenated proteins. VI. Chlorocasein, ibid., 1926, 45, 825. VII. Chlorofibrin, ibid., 1927, 46, 133. VIII. Chlorogluten, ibid,, 1927, 46, 590. IX. Chlorovoprotein, ibid., 1928, 47, 458. 